\title{
Utilising Stochastic Diffusion Search to Identify Metastasis in Bone Scans and Microcalcifications on Mammographs
}

\author{
Mohammad Majid al-Rifaie \\ Department of Computing \\ Goldsmiths, University of London \\ London, SE14 6NW, U.K. \\ Email:m.majid@gold.ac.uk
}

\author{
Ahmed Aber \\ Department of Surgery \\ University of Leicester Royal Infirmary \\ Leicester, LE2 7LX, UK \\ Email:ahmed.aber@nhs.net
}

\author{
Ahmed Majid Oudah \\ Department of Electronic Engineering \\ SRTTU University \\ Tehran, Iran \\ Email: ahmed.majid.oudah@gmail.com
}

\begin{abstract}
This paper introduces a novel approach in using a swarm intelligence algorithm - Stochastic Diffusion Search - as a tool to identify metastasis in bone scans and microcalcifications on the mammographs. This algorithm is adapted for this particular purpose and its performance is investigated by running the agents of the swarm intelligence algorithm on sample bone scans whose status have been determined by the experts. The result of a statistical analysis is also reported, highlighting the behaviour of the algorithm when presented with different samples. Additionally a mathematical approach is presented, providing guideline for estimating the number of iterations required before the swarm intelligence algorithm terminates.
\end{abstract}

\section{INTRODUCTION}

Computer aided diagnosis (CAD) is an emerging field in medicine. The technique introduced in this paper can help radiologists to examine the image in greater depth and has the potential to help doctors from different medical disciplines to interpret medical imaging with greater confidence. Furthermore CAD is a promising learning tool for both medical students and junior doctors to develop basic diagnostic skills. This paper presents a new CAD approach in which a swarm intelligence algorithm - Stochastic Diffusion Search (SDS)[1] - is applied to two medical imaging modalities: the first application is used to detect areas of high technetium-99m-labeled diphosphonates on bone scans and in the second one potential areas of microcalcifications on the $\mathrm{x}$-ray mammography are detected.

Understanding the basics behind the behaviour of the swarm intelligence algorithm and its connection to nature is vital. Communication - social interaction or information exchange - observed in social insects is important in all swarm intelligence algorithms, including Stochastic Diffusion Search (SDS), which mimics the recruitment behaviour of one species of ants - Leptothorax acervorum. Although as stated in [2], in real social interactions, not just the syntactical information is exchanged between the individuals but also semantic rules and beliefs about how to process this information, in swarm intelligence algorithms only the syntactical exchange of information is considered.
There are different forms of recruitment in social insects: it may take the form of local or global, one-to-one or one-tomany, and stochastic or deterministic mode. The nature of information exchange also varies in different environments and with different types of social insects. Sometimes, the information exchange is more complex where, for example, it might carry data about the direction, suitability of the target and the distance; sometimes the information sharing is simply a stimulation forcing a certain triggered action. What all these recruitment and information exchange strategies have in common is distributing useful information in their community.

This paper starts by describing the standard Stochastic Diffusion Search, followed by an introduction to bone scintigraphy, explaining metastatic disease and a brief explanation on how to detect metastasis in bone scans. Afterwards, the swarm intelligence algorithm is adapted for the purpose of this research, the results is reported and a statistical analysis is presented demonstrating the performance of the approach. Next, a brief summary of x-ray mammography and its use is presented, emphasising on mammographic film reading as a particularly demanding visual task, which could be facilitated using the technique presented in this paper.

It is vital to note that the presented approach does not attempt to replace the experts' eyes of radiologists, but rather to aid them in the diagnosis process. The software has been used as an educational tool on several occasions to teach medical students and junior doctors.

\section{Stochastic Diffusion Search}

This section introduces Stochastic Diffusion Search (SDS) [1] - a swarm intelligence algorithm - whose performance is based on simple interaction of agents.

The SDS algorithm commences a search or optimisation by initialising its population and then iterating through two phases (see Algorithm 1)

In the test phase, SDS checks whether the agent hypothesis is successful or not by performing a hypothesis evaluation which returns a boolean value. Later in the iteration, contingent on the precise recruitment strategy employed (in 


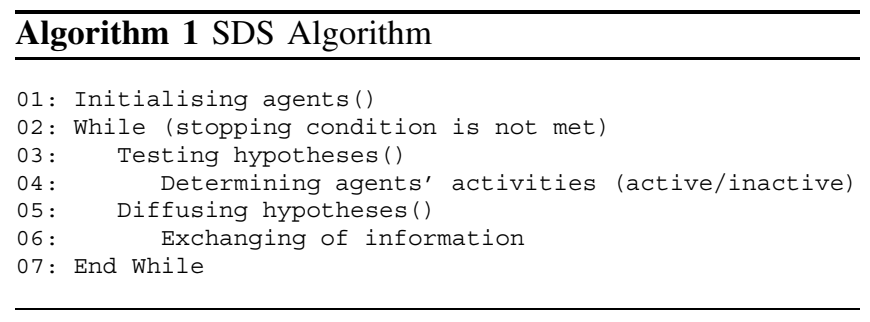

the diffusion phase), successful hypotheses diffuse across the population and in this way information on potentially good solutions spreads throughout the entire population of agents. In other words, each agent recruits another agent for interaction and potential communication of hypothesis. This algorithm has been used alongside other swarm intelligence algorithms in several fields (e.g. [3], [4], [5], [6]).

\section{A. Standard SDS and Passive Recruitment}

In standard SDS (which is used in this paper), passive recruitment mode is employed. In this mode, if the agent is inactive, a second agent is randomly selected for diffusion; if the second agent is active, its hypothesis is communicated (diffused) to the inactive one. Otherwise there is no flow of information between agents; instead a completely new hypothesis is generated for the first inactive agent at random (see Algorithm 2). Therefore, recruitment is not the responsibility of the active agents. In this work, activity of each agent is determined when its fitness is compared against a random agent (which is different from the selecting one); if the selecting agent has a better fitness (smaller value in minimisation problems) than the randomly selected agent, it will be flagged as active, otherwise inactive. Higher rate of inactivity boosts exploration, whereas a lower rate biases the performance towards exploitation.

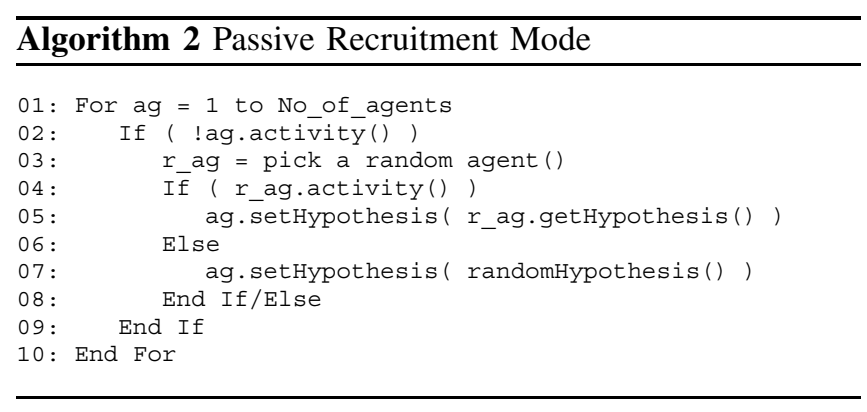

\section{BONE SCINTIGRAPHY}

Bone scan or Bone scintigraphy is one of the most frequently performed of all radionuclide procedures. Radionuclide bone imaging is quick, relatively inexpensive, widely available, exquisitely sensitive and is invaluable in the diagnostic evaluation of numerous pathologic conditions. Although protocols vary among institutions, imaging is typically performed 26 hours after intravenous administration of technetium-99m-labeled diphosphonates. The delay between injection and imaging allows clearance of the radiotracer from the soft tissues, resulting in a higher targetto-background ratio and improved visualization of bone. The degree of radiotracer uptake depends primarily on two factors: blood flow and, perhaps more importantly, the rate of new bone formation [7].

\section{A. Normal Scintigraphic Findings}

There is symmetric distribution of activity throughout the skeletal system in healthy adults. Urinary bladder activity, faint renal activity, and minimal soft-tissue activity are also normally present (see Fig. 1 Top-left).

The accumulation of radiotracer in bone generally decreases with age. However, there are sites of persistently increased symmetric uptake, such as the acromial and coracoid processes of the scapulae, the medial ends of the clavicles, the junction of the body and manubrium of the sternum (angle of Louis), and the sacral alae. Increased radiotracer accumulation in the jaw may be due to dental disease or to malocclusion of dentures.

Symmetric areas of increased calvarial activity occurs in hyperostosis frontalis. In the neck, activity in calcified thyroid cartilage and in the apophyseal joints of the cervical vertebrae in patients with asymptomatic degenerative changes can also be seen.

\section{B. Metastatic Disease}

Metastasis is the process by which the cancer spread from the original site at which it started as a primary tumour to other tissues in the body i.e. Prostate cancer metastasising to the bone tissue.

Many if not most bone scans are performed in patients with a diagnosis of cancer, especially carcinoma of the breast, prostate gland, and lung. Radionuclide bone imaging plays an imporant part in tumor staging and management. This imaging technique is extremely sensitive for detecting skeletal abnormalities, and numerous studies have confirmed that it is considerably more sensitive than conventional radiography for this purpose [8]. About $75 \%$ of patients with malignancy and pain have abnormal bone scintigraphic findings. The usual pattern consists of increased radiotracer deposition in areas of new bone tissue formation in response to the damaging effect of cancer on the bone [8], [9]. The presence of multiple, randomly distributed areas of increased uptake of varying size, shape, and intensity are highly suggestive of bone metastases (see Fig. 1 Topmiddle). Although multiple foci of increased activity may be encountered in other pathologic conditions, it is often possible to distinguish metastatic disease from other entities by analyzing the pattern of distribution of the abnormalities. Traumatic injury, in contrast to metastatic disease, generally manifests as discrete focal abnormalities of similar intensity. 


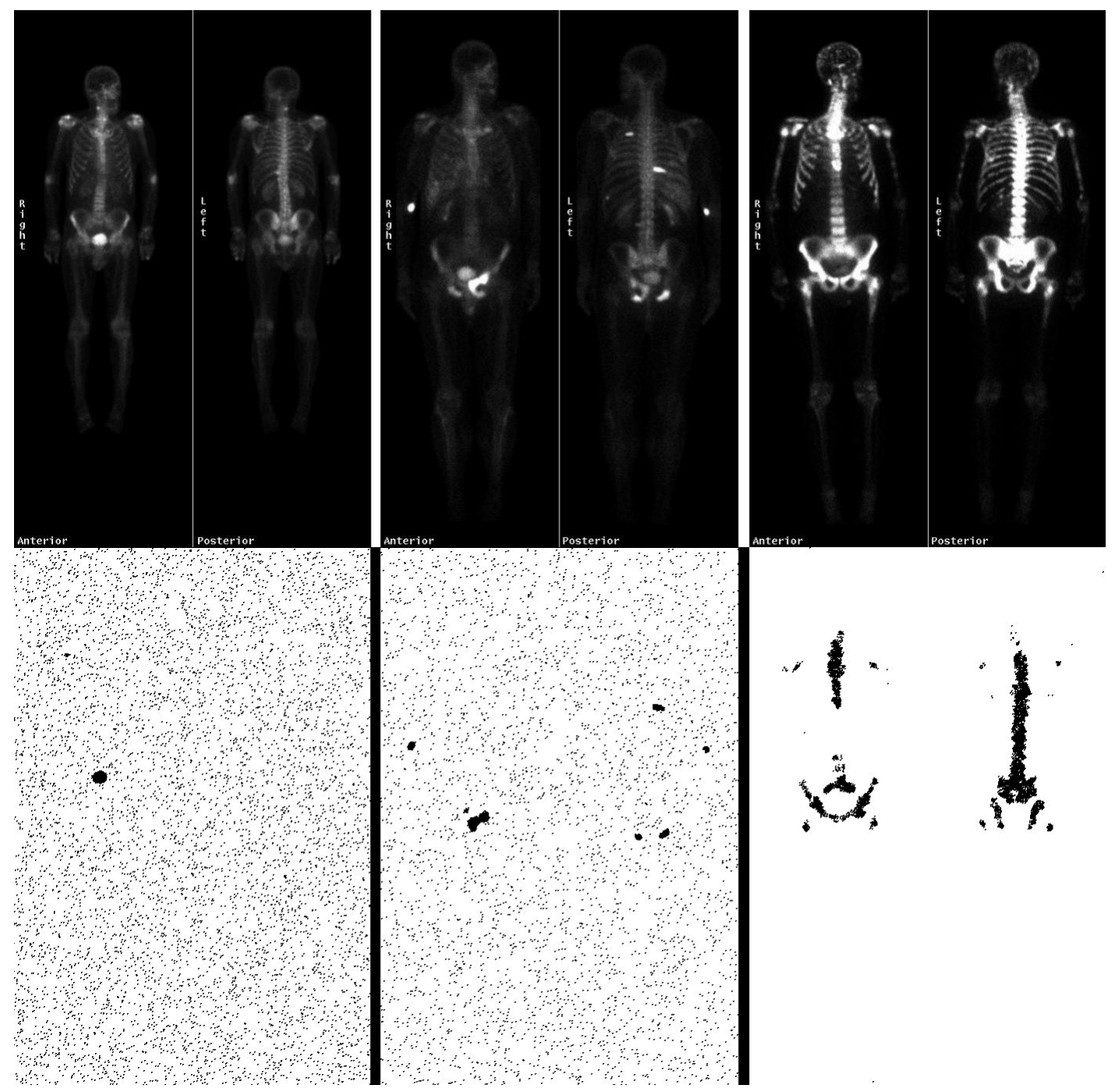

Figure 1. Bone Scans

Top: Typically 2-6 hours after intravenous administration of technetium-99m-labeled diphosphonates; brighter areas indicate a higher radiotracer uptake. Bottom: The scans are processed using Stochastic Diffusion Search algorithm. Left: Healthy; middle: partially affected; right: metastatic disease spread.

In older patients, osteoarthritis and degenerative changes may manifest as areas of intense activity on radionuclide bone images. These changes can be distinguished from metastatic disease by virtue of their characteristic location (e.g. knees, hands and wrists). Involvement of both sides of the joint is common in arthritis but unusual in malignant conditions [10].

When the metastatic process is diffuse, virtually all of the radiotracer is concentrated in the skeleton, with little or no activity in the soft tissues or urinary tract. The resulting pattern, which is characterized by excellent bone detail, is frequently referred to as a superscan (see Fig. 1 Top-right) [9], [10], [11].

Bone scintigraphy is a popular and important imaging modality and will likely remain so for the foreseeable future. Although bone scintigraphy is not specific, its exquisite sensitivity makes it a useful screening procedure for many pathologic conditions, especially for the detection of prostate, breast and lung cancer metastasis.

\section{MAMMOGRAPHY}

$\mathrm{X}$-ray mammography has been shown to be effective as a method for detecting early breast cancer, but the success of mass screening depends critically on the availability of highly skilled film readers to interpret the images. The majority of film readers in the UK are consultant radiologists and in order to maintain a sufficiently high standard of interpretation, readers are required to undergo training, to keep in practice and to evaluate their performance at regular intervals [13].

Mammographic film reading is a particularly demanding visual task. In screening programmes, the film reader must 

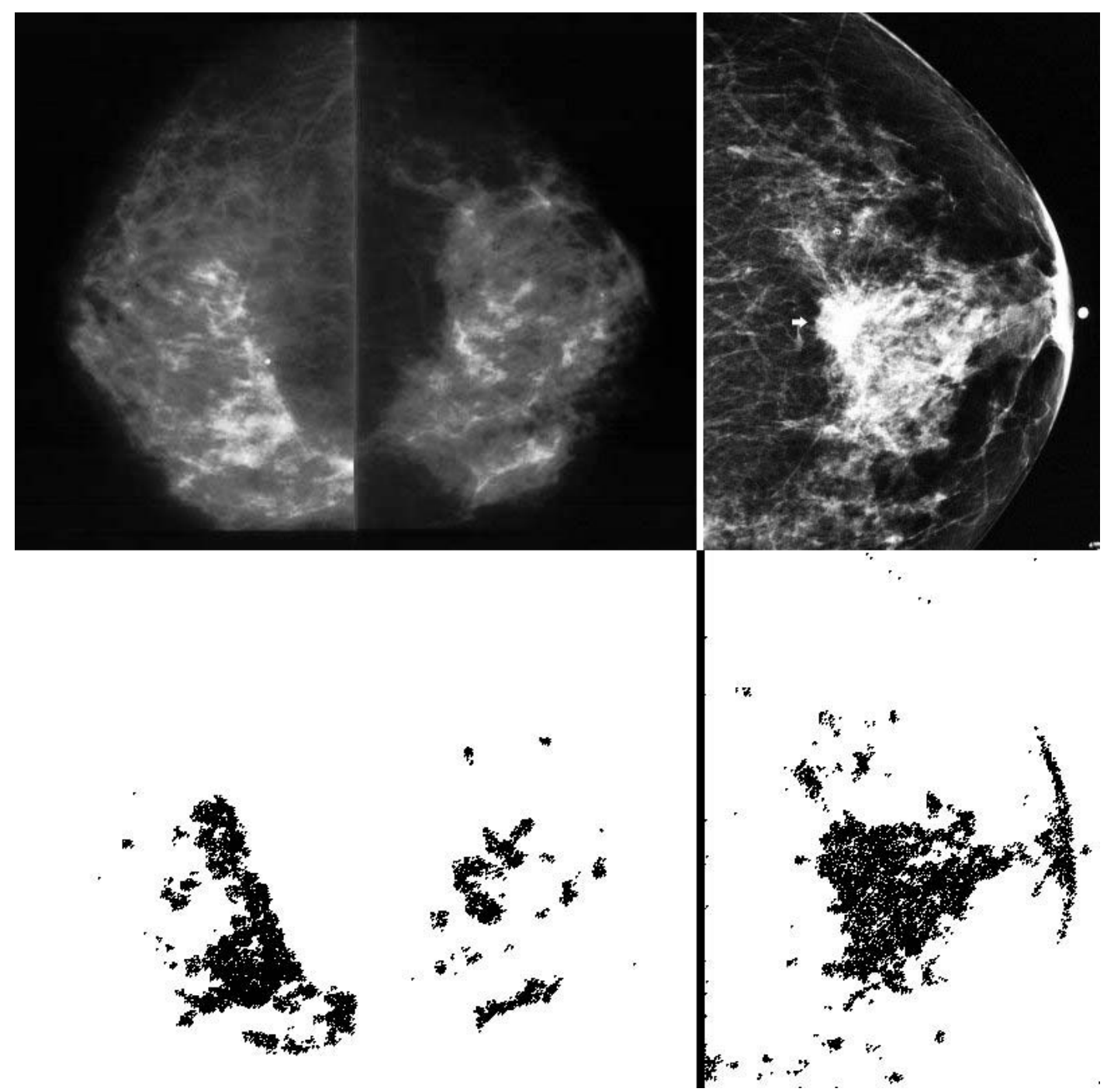

Figure 2. Mammographs

Clusters of microcalcification particles detected using the Stochastic Diffusion Search algorithm.

search for extremely infrequent and often very subtle signs of cancer superimposed on complex and variable backgrounds. Early breast cancer may appear in a variety of forms: a few particles of microcalcification; a small illdefined or speculated mass; abnormal asymmetry between right and left breast images, or subtle distortion of the underlying structure of the breast. These abnormalities vary in size, shape, structure, brightness and location and may share a great deal of similarity with normal mammographic appearances.

False negative cases, in which signs of cancer are missed by a reader, sometimes occur. Retrospective evaluation of the previous screening films of cancers detected between screening rounds (interval cancers) and screen-detected cancers show evidence of abnormality in between $16 \%$ and $27 \%$ of cases. Some of these signs are very subtle, and may have been seen by the readers but dismissed as being insignificant, but others are clear signs of malignancy [14], [15], [16]. However, different readers miss different cancers, as is evidenced by the success of double reading in which two readers independently read the films [17]. The most accurate method of interpretation is double reading with arbitration, where a third reader reviews cases about which the two readers disagree [17], [18].

In the UK particularly with the National Health Service Breast Screening Programme (NHSBSP) there is an increased demand for skilled manpower to effectively interpret mammographs and double or triple reading of the mammograph is not viable option due to the increased workload. A novel and different method of coping with this is the use of computer-based aids.

Researchers have been developing algorithms to detect 


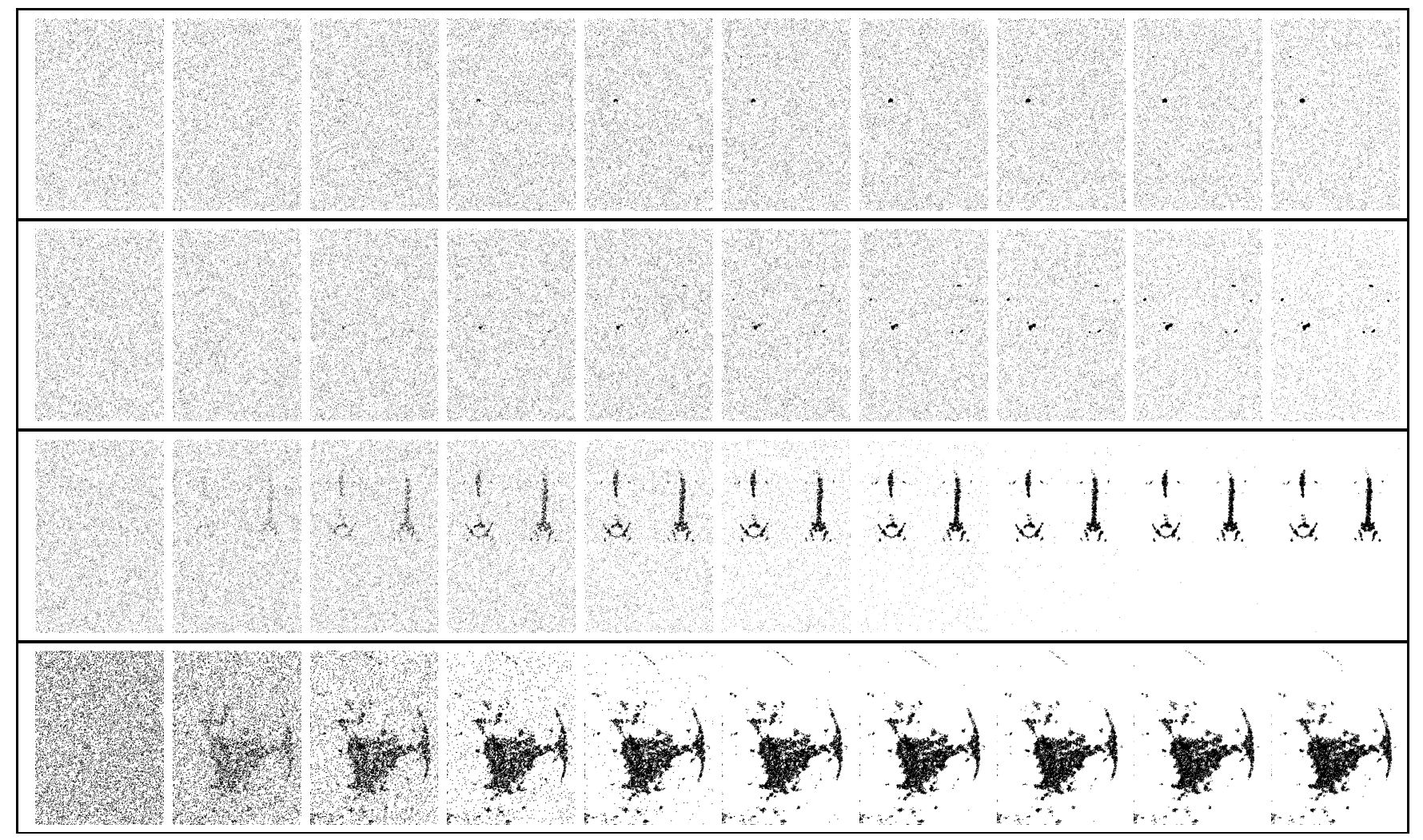

Figure 3. SDS Algorithm Processing Bone Scans and Mammograph in 10 Iterations

Each row shows the behaviour of the agents when presented with one scan. Each scan is processed by 10,000 agents (illustrated as black dots) and through communication, agents explore different areas of the scans to identify potential areas of metastasis and microcalcifications. The leftmost figures in each row show the location of the agents on the first iteration, and the rightmost ones represent the last iteration. Top three rows are bone scans and the bottom row is a mammograph.

mammographic abnormalities for more than 30 years with the aim of either automating mammographic interpretation or, more realistically, providing a tool which will enhance human film-reading performance.

There are two basic approaches to the problem of detecting abnormalities in mammograms: either to search the images for specific appearances suggestive of cancer, or to characterize normal mammographic appearance to the extent that it is possible to detect anything that fails to conform to the generated model of normality.

The purpose of the current study is to apply for the first time an swarm intelligence algorithm namely Stochastic diffusion search to perform the task of identifying the microcalcifications on the mammographs.

\section{Applying Stochastic Diffusion Search}

In this paper, we are presenting a unique approach by deploying SDS to detect the bone metastasis and microcalcifications on the mammographs. This approach demonstrates a promising ability to undertake this task with similar level of sensitivity. Each scan used in this paper is processed by the SDS agents which are responsible for locating the affected areas.

The reproducibility and the accuracy of the SDS algorithm can be utilised in developing a standardised system to interpret bone scans and mammographs preventing operator errors and discrepancies. This technology can be employed as an adjunct to help radiologists assess the various parts of the bone scans and mammographs making the diagnosis of the lesions more thorough and less time consuming. Additionally this technique can be effectively used to develop programs for teaching and training medical students and junior doctors.

\section{A. Experiment Setup}

This section presents the technical details and the experiment setup, followed by the results and a statistical analysis of the performance of the algorithm.

The number of agents used in this experiment is 10,000 and the algorithm is run for 10 iterations (i.e. 10 cycles of test and diffusion phases). As stated earlier in Section II, in the beginning of the process, all the agents are initialised randomly throughout the search space. 


\begin{tabular}{|c|c|c|}
\hline 0 & 0 & 0 \\
\hline 0 & $x$ & 0 \\
\hline$\circ$ & 0 & 0 \\
\hline
\end{tabular}

Figure 4. Agent's Neighbours in Test Phase

The symbol $x$ represents the position of the agent and the o's represent the neighbours used during the test phase.

SDS is a population based stochastic algorithm, adapted here to search for areas of metastasis or calcifications in the feasible solution space. The hypothesis vectors of the population are defined as follows:

$$
x_{i}^{g}=\left[x_{i, 1}^{g}, \ldots, x_{i, D}^{g}\right], i=1,2, \ldots, N P
$$

where $g$ is the current iteration, $D$ is the dimension of the problem space $(D=2)$ and $N P$ is the population size. In the first generation, (when $g=0$ ), the $i^{\text {th }}$ vector's $j^{\text {th }}$ component could be initialised as:

$$
x_{i, j}^{0}=x_{\min , j}+r\left(x_{\max , j}-x_{\min , j}\right)
$$

where $r$ is a random number drawn from a uniform distribution on the unit interval $U(0,1)$, and $x_{\min }, x_{\max }$ are the lower and upper bounds of the $j^{\text {th }}$ dimension, respectively. The inital status of all agents are set to false.

In other words, each agent randomly picks a pixel from the image of the scan. During the test phase of SDS algorithm, each agent's status should be determined. The method used here to set the activity of the agents is to find the average of the colour intensity ${ }^{1}$ (avgIn) of each agent and its neighbours (see Fig. 4). If $\operatorname{avgIn}>\alpha$ the agent is flagged active, otherwise inactive ${ }^{2}$.

During the diffusion phase, each inactive agent randomly selects another agent from the population; if the selected agent is active, the selecting agent adopts the hypothesis (i.e. location) of the active agent and the information sharing takes place. The strategy used for information sharing is to randomly pick an area surrounding the active agent (see Fig. 5). Active agents also check their position by continously picking a random pixel in the neighbourhood; this way, an area which does not have a good enough potential is discarded from one iteration to the next.

\section{B. Results}

As shown in Fig. 1 and 2, areas with higher potential of metastasis and calcifications are identified. In bone scans, other than urinary bladder activity, faint renal activity, and minimal soft-tissue activity which are normally present in the scan (Fig. 1 Bottom-left), the existence of multiple,

\footnotetext{
${ }^{1}$ Colour intensity ( $I n$ ) signifies the brightness of pixels, $0 \leq$ In $\leq 255$.

${ }^{2}$ The value of $\alpha$ is problem-dependent and could be adjusted to increase or decrease the sensitivity of the system. In the experiment conducted here, $\alpha=180$ for bone scans, and $\alpha=120$ for mammographs, which requires a higher level of sensitivity as discussed later.
}

\begin{tabular}{|c|c|c|c|c|}
\hline 0 & 0 & 0 & 0 & 0 \\
\hline 0 & 0 & 0 & 0 & 0 \\
\hline 0 & 0 & $x$ & 0 & 0 \\
\hline 0 & 0 & 0 & 0 & 0 \\
\hline 0 & 0 & 0 & 0 & 0 \\
\hline
\end{tabular}

Figure 5. Diffusion Area

The symbol $\mathrm{x}$ represents the position of the active agent and the o's represent the accessible places during the diffusion phase.

randomly distributed areas of increased uptake of varying size, shape, and intensity are highly suggestive of bone metastases (Fig. 1 Bottom-middle). Additionally as stated before, when the metastatic process is distributed, almost all of the radiotracer congregates in the skeleton, with little or no activity in the soft tissues or urinary tract (see Fig. 1 Bottom-right).

In order to visually present the technique used, Fig. 3 illustrates how agents congregate over the areas of interest over time (i.e. iterations) when fed with the scans as inputs of the algorithm. As the figure shows, successful agents diffuse their positions across the population and this way, information on potentially good solutions spreads throughout the entire population of agents. This process is caused through the recruitment strategy, where each agent recruits another agent for interaction and potential communication of the promising areas. Next, two models are presented to distinctively differentiate between different types of bone scans (e.g. not affected, affected and highly affected).

1) Statistical Model: Here, a statistical analysis, TukeyHSD Test [12], is performed to highlight whether there is a significant difference between the activity of the agents when processing the bone scans. Table I (a) shows the activity rate of the populations over each iteration. Three different samples are used for this analysis: Samples 1,2 and 3 refer to the scans in Fig. 1 (left to right). Table I (b) shows that other than the first iteration where the agents are just initialised, different bone scans would result in significantly different activity rates. This could be used as an indicator, highlighting the difference between various scans and whether they are healthy, partially affected or the metastasis is spread.

2) Mathematical Model: Visualising the data produced in Table I (a) could introduce another method of determining which of the three broad category (healthy, partially affected or the metastasis is spread) the bone scan falls into (see Fig. 6). This model is proposed here to calculate the first and the second derivatives using the following formulas:

$$
\begin{gathered}
f_{i}^{\prime s}=\sigma_{i}^{s}-\sigma_{i-1}^{s} \\
f_{i}^{\prime \prime s}=\sigma_{i}^{s}-2 \times \sigma_{i-1}^{s}+\sigma_{i-2}^{s}
\end{gathered}
$$

where $f^{\prime}$ and $f^{\prime \prime}$ are the first and the second derivatives respectively, $\sigma$ represents the number of active agents, $i$ is 
Table I

Activity Status of Agents Processing Bone Scans

(a) Mean \pm standard deviation of the number of active agents in each iteration is shown (rounded to the nearest number).

\begin{tabular}{lccc}
\hline Itr & Sample 1 & Sample 2 & Sample 3 \\
\hline 0 & $0 \pm 0$ & $0 \pm 0$ & $0 \pm 0$ \\
1 & $5 \pm 2$ & $17 \pm 4$ & $277 \pm 16$ \\
2 & $15 \pm 4$ & $47 \pm 9$ & $763 \pm 37$ \\
3 & $33 \pm 8$ & $100 \pm 18$ & $1602 \pm 76$ \\
4 & $66 \pm 18$ & $201 \pm 31$ & $2991 \pm 137$ \\
5 & $129 \pm 33$ & $379 \pm 51$ & $4992 \pm 188$ \\
6 & $245 \pm 62$ & $697 \pm 84$ & $7260 \pm 198$ \\
7 & $461 \pm 110$ & $1250 \pm 141$ & $8947 \pm 123$ \\
8 & $852 \pm 201$ & $2201 \pm 230$ & $9583 \pm 51$ \\
9 & $1557 \pm 351$ & $3650 \pm 330$ & $9708 \pm 22$ \\
\hline
\end{tabular}

(b) Based on TukeyHSD Test, if the difference between each pair of samples is significant, the pairs are marked ( $\mathrm{o}-\mathrm{X}$ shows that the right sample has significantly more active agents than the left one). This test uses $95 \%$ family-wise confidence level. The aim is to show that agents dealing with scans which have different levels of metastasis exhibit significantly different behaviour.

\begin{tabular}{cccc}
\hline Itr & $\mathbf{s 1}-\mathbf{s 2}$ & $\mathbf{s 1}-\mathbf{s 3}$ & $\mathbf{s 2}-\mathbf{s 3}$ \\
\hline 0 & - & - & - \\
1 & $0-\mathrm{X}$ & $0-\mathrm{X}$ & $\mathrm{o}-\mathrm{X}$ \\
2 & $\mathrm{o}-\mathrm{X}$ & $\mathrm{o}-\mathrm{X}$ & $\mathrm{o}-\mathrm{X}$ \\
3 & $\mathrm{o}-\mathrm{X}$ & $\mathrm{o}-\mathrm{X}$ & $\mathrm{o}-\mathrm{X}$ \\
4 & $\mathrm{o}-\mathrm{X}$ & $\mathrm{o}-\mathrm{X}$ & $\mathrm{o}-\mathrm{X}$ \\
5 & $\mathrm{o}-\mathrm{X}$ & $\mathrm{o}-\mathrm{X}$ & $\mathrm{o}-\mathrm{X}$ \\
6 & $\mathrm{o}-\mathrm{X}$ & $\mathrm{o}-\mathrm{X}$ & $\mathrm{o}-\mathrm{X}$ \\
7 & $\mathrm{o}-\mathrm{X}$ & $\mathrm{o}-\mathrm{X}$ & $\mathrm{o}-\mathrm{X}$ \\
8 & $\mathrm{o}-\mathrm{X}$ & $\mathrm{o}-\mathrm{X}$ & $\mathrm{o}-\mathrm{X}$ \\
9 & $\mathrm{o}-\mathrm{X}$ & $\mathrm{o}-\mathrm{X}$ & $\mathrm{o}-\mathrm{X}$ \\
\hline
\end{tabular}

the iteration number and $s$ is the bone scan sample number, $s=\{1,2,3\}$. The value of the second derivative $\left(f^{\prime \prime}\right)$ can be used as an indicator to stop the algorithm. The rationale behind stopping the algorithm is that the activity of the agents has reached a point that allowing further process, would blur the congregation of the agents around less popular clusters. This is caused by the diffusion mechanism of the algorithm where there is higher probability of an inactive agents picking another one from within the larger clusters than the smaller ones.

One of the main aims of the CAD systems is to identify microcalcifications to help the radiologists make the
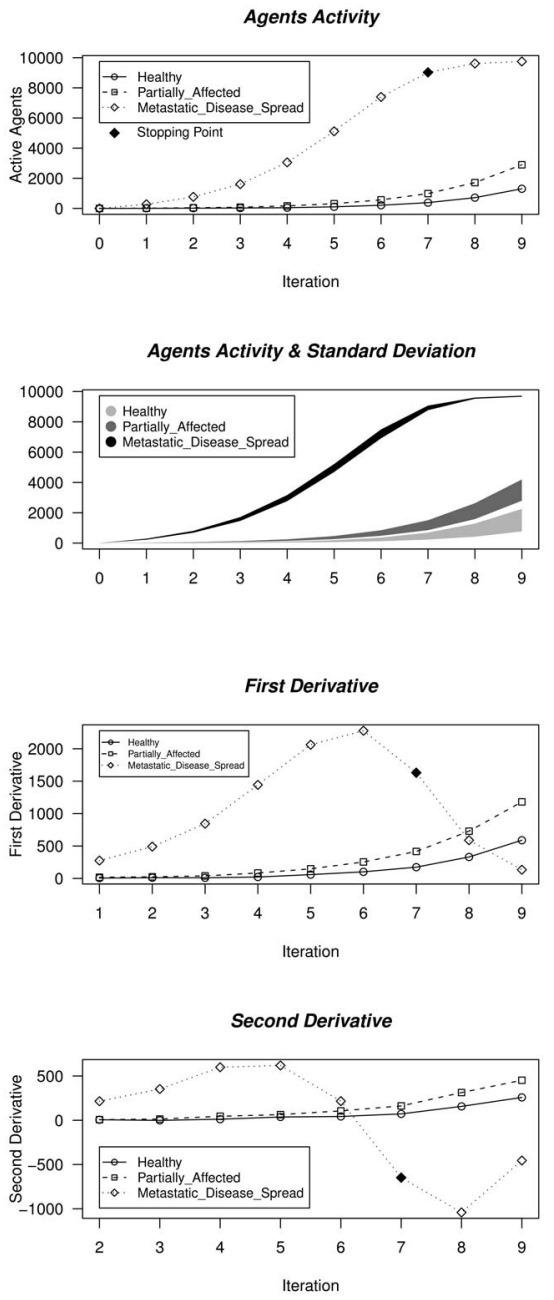

Figure 6. Agents Activity

The plots in this figure illustrate the activity of agents, the standard deviation, first and second derivatives on each iteration in the three sample bone scans. The 'stopping point' in the top plot shows the iteration number when SDS algorithm could terminate. This occurs when the value of the second derivative is negative (see Eq. 4).

diagnosis. Microcalcifications are sometimes difficult for the human film reader to detect because of their small size and low contrast, particularly if they are superimposed on dense glandular tissue. However, of all the signs of abnormality found on mammograms, microcalcifications are the easiest to detect automatically. Unlike small ill-defined masses, which may superficially resemble normal glandular tissue, microcalcifications have properties namely their very small size and high attenuation which differ significantly from those of normal background structures.

Since smaller clusters are of interest in bone scan and specially mammographs, a mechanism for determining the 'stopping point' is proposed. In this method, when $f^{\prime \prime}<0$ the algorithm reaches the stopping point. 


\section{CONCLUSION}

This paper details the promising results of the novel application of Stochastic Diffusion Search in detecting areas of metastasis in bone scans and the identification of the potential microcalcifications on the mammographs. Statistical and mathematical models are proposed to further investigate the behaviour of the agents in the population and the outcome demonstrates that the algorithm exhibits a statistically significant difference when applied to scans of variously affected individuals. Finally the authors would like to emphasise that the presented technique could be effectively utilised as an adjunct to the expert's eyes of a specialist.

\section{REFERENCES}

[1] J. Bishop, "Stochastic searching networks." London, UK: Proc. 1st IEE Conf. on Artificial Neural Networks, 1989, pp. 329-331.

[2] J. F. Kennedy, R. C. Eberhart, and Y. Shi, Swarm intelligence. San Francisco ; London: Morgan Kaufmann Publishers, 2001.

[3] M. M. al-Rifaie, M. Bishop, and T. Blackwell, "An investigation into the merger of stochastic diffusion search and particle swarm optimisation," in GECCO '11: Proceedings of the 2011 GECCO conference companion on Genetic and evolutionary computation. ACM, 2011, pp. 37-44.

[4] M. M. al-Rifaie, A. Aber, and M. Bishop, "Cooperation of nature and physiologically inspired mechanisms in visualisation," in Biologically-Inspired Computing for the Arts: Scientific Data through Graphics, A. Ursyn, Ed. IGI Global, United States, 2012, iSBN13: 9781466609426, ISBN10: 1466609427.

[5] M. M. al-Rifaie, M. Bishop, and S. Caines, "Creativity and autonomy in swarm intelligence systems," in Cognitive Computation: Computational Creativity, Intelligence and Autonomy, M. Bishop and Y. Erden, Eds. Springer, 2012, dOI: 10.1007_s12559-012-9130-y.

[6] M. M. al-Rifaie, M. Bishop, and T. Blackwell, "Resource allocation and dispensation impact of stochastic diffusion search on differential evolution algorithm; in," in Nature Inspired Cooperative Strategies for Optimisation (NICSO 2011), Springer, 2011.

[7] J. McAfee, R. Reba, and M. Majd, "The musculoskeletal system," Principles of Nuclear Medicine. 2nd ed. Philadelphia, PA: WB Saunders, pp. 986-1012, 1995.

[8] W. Hage, A. Aboulafia, and D. Aboulafia, "Incidence, location, and diagnostic evaluation of metastatic bone disease," Orthopedic Clinics of North America, vol. 31, no. 4, pp. 515528, 2000.

[9] C. Bibbo, D. Patel, and J. Benevenia, "Perioperative considerations in patients with metastatic bone disease," Orthopedic Clinics of North America, vol. 31, no. 4, pp. 577-595, 2000.

[10] J. Thrall and H. Ziessman, "Skeletal system," Nuclear Medicine. The Requisites, vol. 2, pp. 110-45, 2001.
[11] M. Weckesser, V. Müller-Mattheis, H. Vosberg, and H. Müller-Gärtner, "Pathogenetic differentiation of the bone superscan using bone marrow scintigraphy]." Nuklearmedizin. Nuclear medicine, vol. 37, no. 4, p. 156, 1998.

[12] R. Miller, "Simultaneous statistical inference," SPRINGERVERLAG INC., 175 FIFTH AVE., NEW YORK, NY, 1981, 300,1981 .

[13] C. Beam, D. Sullivan, and P. Layde, "Effect of human variability on independent double reading in screening mammography," Academic radiology, vol. 3, no. 11, pp. 891-897, 1996.

[14] J. Sumkin and D. Gur, "Computer-aided detection with screening mammography: improving performance or simply shifting the operating point?" Radiology, vol. 239, no. 3, pp 916-918, 2006.

[15] R. Brem, J. Baum, M. Lechner, S. Kaplan, S. Souders, L. Naul, and J. Hoffmeister, "Improvement in sensitivity of screening mammography with computer-aided detection: a multiinstitutional trial," American Journal of Roentgenology, vol. 181, no. 3, pp. 687-693, 2003.

[16] A. Burgess, "On the noise variance of a digital mammography system," Medical physics, vol. 31, pp. 1987-1995, 2004.

[17] E. Burnside, E. Sickles, R. Sohlich, and K. Dee, "Differential value of comparison with previous examinations in diagnostic versus screening mammography," American Journal of Roentgenology, vol. 179, no. 5, pp. 1173-1177, 2002.

[18] D. Chakraborty, "Maximum likelihood analysis of freeresponse receiver operating characteristic (froc) data," Medical physics, vol. 16, p. 561, 1989.

\section{CONTENTS}

I Introduction 1

II Stochastic Diffusion Search

II-A Standard SDS and Passive Recruitment ............ 2

III Bone Scintigraphy 2

III-A Normal Scintigraphic Findings . . . . 2

III-B Metastatic Disease . . . . . . . . . 2

IV Mammography 3

V Applying Stochastic Diffusion Search 5

V-A Experiment Setup . . . . . . . 5

V-B Results ............ 6

V-B1 Statistical Model . . . . . 6

V-B2 Mathematical Model . . . 6

VI Conclusion

References 8 